\title{
A novel score for highly accurate and efficient prediction of native protein
}

2

3

4

5 . Key Laboratory of Bioinformatics, Ministry of Education, School of Life Sciences,

6

7

8

9

10

11

12

13 structures

\author{
Lu-yun Wu, Xia-yu Xia ${ }^{*}$ and Xian-ming Pan ${ }^{*}$
}

*To whom correspondence should be addressed: Dr. Xian-ming Pan, or Dr. Xia-yu Xia School of Life Sciences, Tsinghua University, Beijing, 100084, China, Telephone: +86-10-62792827; E-Mail: pan-xm@mail.tsinghua.edu.cn 


\section{Abstract}

15 Protein structure resolution has lagged far behind sequence determination, as it is often laborious and time-consuming to resolve individual protein structure - more

17 often than not even impossible. For computational prediction, due to the lack of detailed knowledge on the folding driving forces, how to design an energy function is still an open question. Furthermore, an effective criterion to evaluate the performance of the energy function is also lacking. Here we present a novel knowledge-based-energy scoring function, simply considering the interactions of

22 peptide bonds, rather than, as conventionally, the residues or atoms as the most 23 important energy contribution. This energy scoring was evaluated by selecting the $\mathrm{X}$-ray structure from a large number of possibilities. It not only outperforms the best of the previously published statistical potentials, but also has very low computational expense. Besides, we suggest an alternative criterion to evaluate the performance of 27 the energy scoring function, measured by the template modeling score of the selected rank-one. We argue that the comparison should allow for some deviation between the

29 X-ray and predicted structures. Collectively, this accurate and simple energy scoring function, together with the optimized criterion, will significantly advance the computational protein structure prediction. 
A protein's structure determines its thermodynamic and biological function, and provides the three-dimensional basis for drug design ${ }^{1-3}$. However, the resolution of protein structures lags far behind their sequence determination, since the resolution often is laborious, time-consuming, and sometimes impossible ${ }^{4}$. To facilitate the resolution process, computational prediction methods have been developed. However, the accuracy of the predicted structures is still being questioned ${ }^{5-7}$.

The total size of the conformational landscapes increased exponentially with the increasing of the protein chain length and very quickly become astronomical ${ }^{8}$. Designing an accurate and simple energy scoring function is a crucial task in protein structure prediction. Several physics- and knowledge-based energy functions have been developed. Physical energy functions such as CHARMM, AMBER, GROMOS, and OPLS $^{9-12}$ are widely used in areas such as protein structure refinement. However, they have limited application in protein structure prediction, due to the computational expense and lack of significant gains ${ }^{13,14}$. As an alternative to physics-based energy functions, knowledge-based energy functions have been widely employed in protein structure prediction ${ }^{15-18}$. However, according to the CASP, no method is able to predict the structures of all kinds of proteins. Here, we assume that the interactions between a protein's peptide bonds, rather than as conventionally, the residues or atoms are the most important energy contribution. We design a novel energy scoring function, named PBE scoring function. The PBE scoring function not only outperforms the tops of the previously published energy scoring function, but also has a factor of 39 to 350 lower CPU time requirement. Additionally, the parameters of the 
PBE scoring function were obtained from fitting an in-house dataset, where each protein has a sequence identity less than $20 \%$ with that in all test datasets used to avoid over-fit problem. Our results suggest that peptide bond is much more suitable for constructing knowledge-based energy functions than residues or atoms. This energy function should advance the computational protein structure prediction and highlight the importance of the interactions between peptide bonds as the protein folding driving force.

Another challenge for computational structure prediction is to identify when the prediction is correct. Currently, the predicted structure is considered correct only if it exactly matches the experimentally determined x-ray structure. However, we believe this criterion is unjustified: Often, we find that predictions are classified as erroneous when (1) there is uncertainty in the x-ray structure, or (2) the difference between the $\mathrm{x}$-ray and the predicted structures is so small that the two structures are identical for all practical purposes. Thus, we propose a novel criterion that takes these challenges into account. With a structural-similarity threshold TM-score $>0.7$, the PBE scoring function achieves a success rate of $100 \%$ on the CASP5-8 dataset

In the following, we will first present the design of the PBE energy function and the PBE's prediction performance with the conventional criterion. Then, we present the new criterion, before re-evaluating the PBE's prediction performance with the new criterion.

\section{Design of the PBE function}


To choose the native structures from a large number of decoys, we design a new protein PBE scoring function. We focus on the peptide bond, since proteins are composed of amino acids or residues joined together by peptide bonds. Note that the peptide bond has a slight double-bond character (40\%). Due to resonance, which occurs with amides, the $\mathrm{C}-\mathrm{N}$ bond length is $10 \%$ shorter than that found in usual C-N amine bonds. Furthermore, $\mathrm{C}_{\alpha}, \mathrm{C}$ and $\mathrm{O}$ atoms of the $i$ th residue, as well as $\mathrm{C}_{\alpha}, \mathrm{N}$ and $\mathrm{H}$ atoms of the $(i+1)$ th residue, are approximately coplanar. This rigidity of the peptide bond reduces the degrees of freedom of the polypeptide during folding. As a consequence, all peptide bonds in protein structures are found to be almost planar. This planar characteristic of the peptide bond constrains the protein rotation, which finally shapes the overall three-dimensional protein structure. The interaction with one residue (or atom) within a peptide bond would be conducted to all groups within this peptide due to the resonance effect, indicating that the interaction with the group within a peptide bond is nearly equivalent to the interaction with the entire peptide bond. For these reasons, our method focuses on the protein peptide bonds and their directions, in which the proteins' peptide bonds are considered as the structural entities,

In a protein structure, the $i$ th residue is represented by the coordinate vector $D_{i}$ of its $C_{\alpha}$-atom, and then its peptide bond direction is defined by the vector $V_{i}$ $\left(V_{i}=D_{i+1}-D_{i}\right)$. The interaction between the $i$ th and $j$ th peptide bonds is represented by the cosine value $\left(\cos (\theta): \theta\right.$ is the angle between $V_{i}$ and $\left.V_{j}\right)$. To calculate the PBE score of the $i$ th peptide bond, we consider 3 adjoining peptide bonds, namely, $i-1, i$ 

and $i+1$, and assume that it is affected by both localized and distant interactions The PBE score of a peptide bond is defined as:(eq. 1):

$$
P B E=\sum_{i=1}^{3 * 4} \sum_{j=1}^{20} \alpha_{i, j}{ }^{L} P_{i}{ }^{\prime}(j)+\sum_{i=1}^{3^{*} 4} \sum_{j=1}^{20} \beta_{i, j}{ }^{S} P_{i}{ }^{\prime}(j)
$$

The PBE score of a structure is defined as the average of all peptide bonds of the structure. In equation 1, the first term on the right hand side describes the long-range peptide interactions, while the second term denotes the short-range peptide interactions.

The long-range term represent the interactions between the peptide bonds in the window, and the spatially closest bonds but in sequence outside the window. Three long-range interactions occur for each peptide bond in the window, producing $3 * 4=12$ new PSSM matrix vectors ${ }^{{ }}{ }_{P} \cdot$, where $\alpha_{\mathrm{i}, \mathrm{j}}$ denotes the coefficients. The second item represents the short-range interaction between the three peptide bonds in the window. Three short-range interactions also exist, producing $3 * 4=12$ new PSSM matrix vectors $s_{P_{i}}$, , where $\beta_{i, j}$ denotes the coefficients. In total, 480 coefficients exist in the energy scoring function.

We assume that the interaction between two peptide bonds leads to a change in the values of the PSSM matrix vector $P$ of the four residues included in these two peptide bonds.

$$
\begin{aligned}
& P_{i}^{\prime}=\cos (\theta) P_{j}+P_{i} \\
& P_{i+1}^{\prime}=\cos (\theta) P_{j+1}+P_{i+1}
\end{aligned}
$$




$$
\begin{aligned}
& P_{j}^{\prime}=\cos (\theta) P_{i}+P_{j} \\
& P_{j+1}^{\prime}=\cos (\theta) P_{i+1}+P_{j+1}
\end{aligned}
$$

Furthermore, we classify the PBE score function into $8 * 8 * 8=512$ categories according to the cosine values of the short-range interactions. In each category, the samples of the in-house dataset can be used to set up more than 600000 equations to fit 480 parameters. This large number of training samples ensures the validity of parameters and avoids the over-fitting problem.

All the coefficients are determined by the in-house training data set using a multiple linear regression method to minimize the sum of the square of the deviations between the left and the right side of the equation (1) ${ }^{19}$. During training, for each peptide bond, the PBE score on the left side of equation (1) is set to 0.0 for an $\mathrm{x}$-ray structure and to 1.0 - TM-score for a decoy structure. The prediction is performed with "Jackknife" analysis ${ }^{19}$, exploiting the database. Each of the sample proteins in the database (including the $\mathrm{x}$-ray structure and decoys) is excluded from the calculation of the coefficients. These coefficients are used in equation (1) to calculate the PBE score of the excluded proteins. Under the self-consistent validation, the PBE function score correctly chooses all 5147 x-ray structures as having the lowest free energy, achieving a success rate of $100 \%$ and a Z-score of -9.67 . When we perform the jackknife test, 5070 protein X-ray structures are correctly identified as having the lowest free energy. This performance equals a success rate of $98.62 \%$ and a Z-score of -9.01 (Table 1 in extended data). The success rate decreases only by $1.4 \%$ under the 
139 jackknife test, suggesting that the score is quite robust.

140

141

142

143

144

145

146

147

148

149

150

151

152

153

154

155

156

157

158

159

\section{Performance on conventional criterion}

To measure the performance of the PBE function, we try to predict the exact x-ray structures in the widely used CASP5-8 dataset. This dataset contains 2628 possible structures (i.e. decoys) for 143 proteins. The PBE function assigns the lowest free energy to the exact x-ray structure for 122 of the $143(85 \%)$ proteins, and achieves a Z-score of -2.84 . The $85 \%$ and the Z-score are both higher than for all 32 considered, previously published energy functions (table 1). The next highest performance is achieved by the functions RW, RWplus, dDFIRE, and KBF, which identified 110(77\%), 106(74\%), 100(70\%) and 97(68\%) x-ray structures respectively. Also the cost of CPU time of PBE is significantly lower than all the previously published functions. E.g., the cost of CPU time on the CASP5-8 dataset is 19 seconds for the PBE, while 743 seconds for dDFIRE, 915 seconds for RW, 2010 seconds for the RWplus and 6701 seconds for KBF. Thus, the PBE constitutes a significant improvement in both computational expense reduction and accuracy improvement.

\section{The novel evaluation criterion}

Our second contribution, to the improvement of computational protein structure prediction, is the novel prediction evaluation criterion. To develop this criterion, we investigate the reason why the PBE's predictions were classified as failures for 21 proteins in the CASP8-5 dataset. For this prediction, we employ the criterion where we require the exact match with the x-ray structure. Thus, we investigate the 
160

161

162

163

164

165

166

167

168

169

170

171

172

173

174

175

difference between the $21 \mathrm{x}$-ray and the 21 PBE predicted structures: In detail, we evaluate these structural differences with the template modeling score (TM-score $)^{20}$, as the TM-score measures the global similarity of two protein structures and is length-independent. According to Zhang, et al. ${ }^{21}$, two protein structures with a TM-score $>0.5$ are likely to have the same topology, while those with a score $>0.8$ certainly have the same topology. Interestingly, when the TM-score is greater than 0.9 , both Zhang and $\mathrm{Hrabe}^{22}$, et al. suggest that it is unnecessary to distinguish the predicted structures from their corresponding x-ray structures, since these two structures are highly likely to be identical. Furthermore, when the TM-score between predicted and $\mathrm{x}$-ray structure is above 0.5 to $0.7^{21}$, the predicted structure is sufficiently accurate to be used in drug design. Within the 21 proteins where the PBE prediction was classified as an erroneous when requiring the exact $\mathrm{x}$-ray structure, there are 6 structures with a TM-score greater than $0.9,9$ structures with a TM-score between 0.8 and 0.9 , and 6 structures with a TM-score between 0.7 and 0.8 . Thus, there are 6 proteins where the predicted structure is, for all practical purposes, identical with the $\mathrm{x}$-ray structure $(\mathrm{TM}$ score $>0.9)$. For these 6 proteins, we still judge the PBE to have failed since we require the exact match between predicted and x-ray structure. Moreover, all the 21 structure predictions would have been sufficient for use in drug design, as their TM scores were above 0.7 , but they were still classified as erroneous. Thus, by requiring the exact match, insignificant differences to the x-ray structure can lead to the prediction being classified as a failure. In our opinion, these predictions should not necessarily have been classified as a failure since they were 
182

183

either virtually identical to the $\mathrm{x}$-ray structure (TM score $>0.9)$ or accurate enough for some task (TM score $>0.7)$. This motivates us to shift away from requiring the exact match with the $\mathrm{x}$-ray structure as the prediction criterion.

Another reason not to require the exact match between the predicted and x-ray structure is that there can be experimental uncertainty in the $\mathrm{x}$-ray determined structure: E.g., Hrabe, et. al. reported that the root mean square deviation (RMSD) between $x$-ray structures of the same protein may range from $0-4 \AA^{22}$, when the structure is determined by different groups. These 0-4 $\AA$ stem from differences in apo and holo forms, physicochemical- condition variations during crystallization, range of the conformational ensemble of the individual protein, etc.. Even, it is possible that the x-ray-determined structure is less similar to the native structure than a predicted structure $^{23}$. Figure 1 shows the relationship between the TM-score and the average RMSD of the high-resolution training set ${ }^{24}$. At a TM-score of approximately 0.7 , the average RMSD is approximately $3 \AA$, which decreases with increasing TM-score. At a TM-score of approximately 0.9 , the average RMSD value is approximately $2 \AA$, indicating that structures with TM-scores greater than 0.9 have the same topology as that of the X-ray structure. We further investigate this $\mathrm{x}$-ray structure uncertainty and the divergence with the predicted structures, by using MaxCluster ${ }^{25}$. MaxCluster aligns the 21 predicted with the $21 \mathrm{x}$-ray structures. When aligned, there is a weak correlation between the temperature factors (B-factor) (Figure 2 presents 3 examples; extended data Figure 1 presents all 21 proteins) and their corresponding $C_{\alpha}$ RMSD values. Thus, in some local regions where the B-factors are high, the $C_{\alpha}$ RMSD values 
204

205

206

207

208

209

210

211

212

are also high. I.e., the correlation between high B-factor and high RMSD value suggest that the $\mathrm{x}$-ray and predicted structures mainly diverge in the region where the protein is flexible. In the areas where the protein is not flexible, the prediction exactly matches the x-ray structure. Indeed, x-ray structure determination of a flexible protein can be difficult, as the x-ray method generally detects the average conformation of proteins, which may not have the lowest energy ${ }^{6,26,27}$. Thus, our analysis of the 21 proteins and the literature agree, that requiring the exact match with the $\mathrm{x}$-ray structure suffers from experimental uncertainty in the x-ray structure.

These insights suggests that considering a prediction to be correct, only when there is an exact match between a predicted and an x-ray structure is not justifiable and unnecessary. For this reason, we decide to develop a new criterion to evaluate when a prediction can be considered to be correct.

Based on literature, we propose a new criterion where the TM-score between the predicted and the x-ray structures is used to evaluate the performance of the energy function. In detail, our novel evaluation criterion calculates the TM-score between the predicted structure with the lowest energy (rank one) and the x-ray structures. If this TM-score is above a certain threshold, the prediction is considered to be correct. Furthermore, the threshold is goal-oriented: If a virtually identical structure is required, the threshold could be 0.9 , or if the prediction is used in drug design, the threshold could be 0.7 , at which the average RMSD between predicted and x-ray structure is approximately $3 \AA$ (Figure 1 ). 
225

226

227

\section{Performance on the novel criterion}

We present the PBE performance on five data sets, when we use the novel TM-score criterion (TM-score $>0.7$; table 2): On the CASP5-8, high resolution, and Rosetta (3D-Robot) datasets, the PBE achieves a success rate of 100\%. Furthermore, on the Modeller_set (3d-robot) and I-TASSER_set (3d-robot) datasets, the success rates are $94.74 \%$ (only one of the predicted structures has a TM score of 0.66 ) and $97.44 \%$ (only one of the predicted structures has a TM score of 0.66 ), respectively.

Overall, with the novel criterion, the PBE can be considered to be highly accurate.

\section{Conclusion}

To increase the accuracy of protein structure prediction, which is the key for its wider applications in the future, we present two significant improvements to the computational protein structure prediction. The first contribution is a novel knowledge-based Peptide-Bond-Interactions-based Energy (PBE) scoring function which predicts the lowest-energy structure from a set of possible (decoy) structures.

The PBE scoring function not only outperforms the most-accurate, previously published statistical potential function, but also has much lower computational expense. In particular, the lower computational expense widens the PBEs potential usage. The second contribution is a novel criterion to evaluate the energy scoring function performance based on similarity with the x-ray structure instead of an exact match. This criterion is based on two realizations: (1) insignificant differences between predicted and x-ray structures lead to a prediction being classified as a failure, 
246 and (2) that the $\mathrm{x}$-ray structure only represents an average native protein structure, due

247 to the flexible nature of the protein structure. Using a combination of these two

248 improvements, we achieved a $99.45 \%$ prediction success rate on all test datasets. Key

249 to achieving the above results is to realize the importance of the peptide bond in the

250 design of the protein energy function. Considering only the peptide bond is a good

251 trade-off between prediction accuracy and computational expense. 


\begin{tabular}{|c|c|c|}
\hline 53 & & \\
\hline 54 & 1 & $\begin{array}{l}\text { Rosenbaum, D. M., Rasmussen, S. G. \& Kobilka, B. K. The structure and function of } \\
\text { G-protein-coupled receptors. Nature } 459,356-363 \text {, doi:10.1038/nature } 08144 \text { (2009). }\end{array}$ \\
\hline 257 & 2 & $\begin{array}{l}\text { Saikrishnan, K. et al. Structure of Mycobacterium tuberculosis single-stranded DNA-binding } \\
\text { protein. Variability in quaternary structure and its implications. J Mol Biol 331, 385-393, } \\
\text { doi:10.1016/s0022-2836(03)00729-0 (2003). }\end{array}$ \\
\hline D & 3 & $\begin{array}{l}\text { Westwood, I. et al. Insights into the Conformational Variability and Regulation of Human } \\
\text { Nek2 Kinase. Journal of Molecular Biology 386, 476-485, doi:10.1016/j.jmb.2008.12.033 } \\
\text { (2009). }\end{array}$ \\
\hline & 4 & $\begin{array}{l}\text { Rupp, B. \& Wang, J. Predictive models for protein crystallization. Methods 34, 390-407, } \\
\text { doi:10.1016/j.ymeth.2004.03.031 (2004). }\end{array}$ \\
\hline & 5 & $\begin{array}{l}\text { Chain, B. M. \& Myers, R. Variability and conservation in hepatitis B virus core protein. Bmc } \\
\text { Microbio/ 5, doi:10.1186/1471-2180-5-33 (2005). }\end{array}$ \\
\hline & 6 & $\begin{array}{l}\text { Bakan, A. et al. Evol and ProDy for bridging protein sequence evolution and structural } \\
\text { dynamics. Bioinformatics 30, 2681-2683, doi:10.1093/bioinformatics/btu336 (2014). }\end{array}$ \\
\hline 3 & 7 & $\begin{array}{l}\text { Lopes, P. E. M., Guvench, O. \& MacKerell, A. D. Current Status of Protein Force Fields for } \\
\text { Molecular Dynamics Simulations. Molecular Modeling of Proteins: 2nd Edition 1215, 47-71, } \\
\text { doi:10.1007/978-1-4939-1465-4_3 (2015). }\end{array}$ \\
\hline & 8 & $\begin{array}{l}\text { Kuhlman, B. \& Bradley, P. Advances in protein structure prediction and design. Nat Rev Mol } \\
\text { Cell Biol 20, 681-697, doi:10.1038/s41580-019-0163-x (2019). }\end{array}$ \\
\hline & 9 & $\begin{array}{l}\text { Brooks, B. R. et al. CHARMM: the biomolecular simulation program. J Comput Chem 30, } \\
\text { 1545-1614, doi:10.1002/jcc.21287 (2009). }\end{array}$ \\
\hline & 10 & $\begin{array}{l}\text { Wang, J. M., Wolf, R. M., Caldwell, J. W., Kollman, P. A. \& Case, D. A. Development and } \\
\text { testing of a general amber force field. J Comput Chem 25, 1157-1174, doi:DOI } \\
\text { 10.1002/jcc. } 20035 \text { (2004). }\end{array}$ \\
\hline & 11 & $\begin{array}{l}\text { Van der Spoel, D. et al. GROMACS: Fast, flexible, and free. J Comput Chem 26, 1701-1718, } \\
\text { doi:10.1002/jcc.20291 (2005). }\end{array}$ \\
\hline & 12 & $\begin{array}{l}\text { Jorgensen, W. L., Maxwell, D. S. \& TiradoRives, J. Development and testing of the OPLS } \\
\text { all-atom force field on conformational energetics and properties of organic liquids. J Am } \\
\text { Chem Soc 118, 11225-11236, doi:DOI 10.1021/ja9621760 (1996). }\end{array}$ \\
\hline 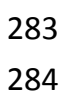 & 13 & $\begin{array}{l}\text { Freddolino, P. L., Park, S., Roux, B. \& Schulten, K. Force Field Bias in Protein Folding } \\
\text { Simulations. Biophys J 96, 3772-3780, doi:10.1016/j.bpj.2009.02.033 (2009). }\end{array}$ \\
\hline 7 & 14 & $\begin{array}{l}\text { Wang, Y. H., Zhang, H., Li, W. \& Scott, R. A. Discriminating Compact Nonnative Structures } \\
\text { from the Native Structure of Globular-Proteins. P Natl Acad Sci USA 92, 709-713, doi:DOI } \\
\text { 10.1073/pnas.92.3.709 (1995). }\end{array}$ \\
\hline J & 15 & $\begin{array}{l}\text { Mishra, A., Iqbal, S. \& Hogue, M. T. Discriminate protein decoys from native by using a } \\
\text { scoring function based on ubiquitous Phi and Psi angles computed for all atom. J Theor Biol } \\
398,112-121 \text {, doi:10.1016/j.jtbi.2016.03.029 (2016). }\end{array}$ \\
\hline ? & 16 & $\begin{array}{l}\text { Feng, Y. P., Kloczkowski, A. \& Jernigan, R. L. Four-body contact potentials derived from two } \\
\text { protein datasets to discriminate native structures from decoys. Proteins-Structure Function } \\
\text { and Bioinformatics } 68,57-66 \text {, doi:10.1002/prot. } 21362 \text { (2007). }\end{array}$ \\
\hline & 17 & $\begin{array}{l}\text { Huang, S. Y. \& Zou, X. Statistical mechanics-based method to extract atomic } \\
\text { distance-dependent potentials from protein structures. Proteins 79, 2648-2661, } \\
\text { doi:10.1002/prot.23086(2011). }\end{array}$ \\
\hline
\end{tabular}


18 Lu, H. \& Skolnick, J. A distance-dependent atomic knowledge-based potential for improved protein structure selection. Proteins-Structure Function and Genetics 44, 223-232, doi:DOI 10.1002/prot.1087 (2001).

19 Xia, X. Y., Ge, M., Wang, Z. X. \& Pan, X. M. Accurate Prediction of Protein Structural Class. Plos One 7, doi:ARTN e3765310.1371/journal.pone.0037653 (2012).

20 Zhang, Y. \& Skolnick, J. Scoring function for automated assessment of protein structure template quality. Proteins-Structure Function and Bioinformatics 57, 702-710, doi:10.1002/prot.20264 (2004).

$21 \mathrm{Xu}$, J. R. \& Zhang, Y. How significant is a protein structure similarity with TM-score=0.5? Bioinformatics 26, 889-895, doi:10.1093/bioinformatics/btq066 (2010).

22 Hrabe, T. et al. PDBFlex: exploring flexibility in protein structures. Nucleic Acids Res 44, D423-D428, doi:10.1093/nar/gkv1316 (2016).

23 Rashin, A. A., Rashin, A. H. L. \& Jernigan, R. L. Protein flexibility: coordinate uncertainties and interpretation of structural differences. Acta Crystallogr $D$ 65, 1140-1161, doi:10.1107/S090744490903145x (2009).

24 Rajgaria, R., McAllister, S. R. \& Floudas, C. A. Distance dependent centroid to centroid force fields using high resolution decoys. Proteins-Structure Function and Bioinformatics 70, 950-970, doi:10.1002/prot.21561 (2008).

25 Siew, N., Elofsson, A., Rychlewski, L. \& Fischer, D. MaxSub: an automated measure for the assessment of protein structure prediction quality. Bioinformatics 16, 776-785, doi:10.1093/bioinformatics/16.9.776 (2000).

26 Monroe, L., Terashi, G. \& Kihara, D. Variability of Protein Structure Models from Electron Microscopy. Structure 25, 592-+, doi:10.1016/j.str.2017.02.004 (2017).

27 Bakan, A., Meireles, L. M. \& Bahar, I. ProDy: protein dynamics inferred from theory and experiments. Bioinformatics 27, 1575-1577, doi:10.1093/bioinformatics/btr168 (2011). 
325 Table 1. Comparison of the $P B E$ with other energy function scores.

\begin{tabular}{|c|c|c|c|c|}
\hline Function & Rank $\leq 5$ & Rank $=1$ & z-score & $\begin{array}{c}\text { Cost of } \\
\text { CPU-time/s } \\
\end{array}$ \\
\hline PBE & 140 & 122 & -2.84 & 19 \\
\hline KBF & 138 & 97 & -1.92 & 6701 \\
\hline RW & 136 & 110 & -1.69 & 915 \\
\hline RWplus & 135 & 106 & -1.69 & 2010 \\
\hline 4BOPT & 131 & 72 & -1.46 & 1 \\
\hline dDFIRE & 128 & 100 & -1.41 & 743 \\
\hline SKOb & 125 & 48 & -1.45 & 1 \\
\hline fourBody & 124 & 53 & -1.30 & 1 \\
\hline SKOa & 122 & 49 & -1.27 & 1 \\
\hline SKJG & 120 & 55 & -1.37 & 1 \\
\hline 4BOPT_GNM & 119 & 46 & -1.06 & 1 \\
\hline MJ3 & 114 & 46 & -1.36 & 1 \\
\hline HLPL & 113 & 50 & -1.28 & 1 \\
\hline Qp & 112 & 57 & -1.32 & 1 \\
\hline BFKV & 112 & 57 & -1.52 & 1 \\
\hline MS & 112 & 42 & -1.34 & 1 \\
\hline VD & 111 & 53 & -1.37 & 1 \\
\hline TEs & 111 & 46 & -1.39 & 1 \\
\hline Qm & 111 & 42 & -1.21 & 1 \\
\hline Qa & 111 & 37 & -1.16 & 1 \\
\hline genFour & 110 & 53 & -1.25 & 1 \\
\hline Tel & 108 & 46 & -1.36 & 1 \\
\hline shortRange & 108 & 39 & -1.05 & 1 \\
\hline BT & 106 & 58 & -1.49 & 1 \\
\hline MJ3h & 106 & 51 & -1.34 & 1 \\
\hline TD & 105 & 57 & -1.30 & 1 \\
\hline GKS & 103 & 38 & -1.18 & 1 \\
\hline MJPL & 100 & 45 & -1.02 & 1 \\
\hline MJ2h & 96 & 45 & -1.05 & 1 \\
\hline TS & 95 & 36 & -1.00 & 1 \\
\hline MSBM & 61 & 17 & -0.11 & 1 \\
\hline RO & 57 & 7 & -0.36 & 1 \\
\hline MJ1 & 14 & 0 & 0.76 & 1 \\
\hline
\end{tabular}

326 /: not calculation 
Table 2. Percentage prediction success rate of the different TM-score threshold on

329 independent datasets.

\begin{tabular}{|c|c|c|c|c|c|}
\hline $\begin{array}{c}\text { TM- } \\
\text { score }\end{array}$ & Casp5-8 & $\begin{array}{c}\text { High } \\
\text { resolution }\end{array}$ & $\begin{array}{l}\text { Rosetta_- } \\
\text { set(3DR) }\end{array}$ & $\begin{array}{c}\text { Modeller_- } \\
\text { set(3DR) }\end{array}$ & I-TASSER_set(3DR) \\
\hline $1.0(x-$ ray $)$ & $85.3 \%$ & $96.39 \%$ & $87.93 \%$ & $68.42 \%$ & $76.92 \%$ \\
\hline$>0.9$ & $89.51 \%$ & $97.20 \%$ & $98.28 \%$ & $84.21 \%$ & $92.31 \%$ \\
\hline$>0.8$ & $95.80 \%$ & $97.20 \%$ & $100 \%$ & $94.74 \%$ & $97.44 \%$ \\
\hline$>0.7$ & $100 \%$ & $100 \%$ & $100 \%$ & $94.74 \%$ & $97.44 \%$ \\
\hline$>0.6$ & $100 \%$ & $100 \%$ & $100 \%$ & $100 \%$ & $100 \%$ \\
\hline$>0.5$ & $100 \%$ & $100 \%$ & $100 \%$ & $100 \%$ & $100 \%$ \\
\hline
\end{tabular}




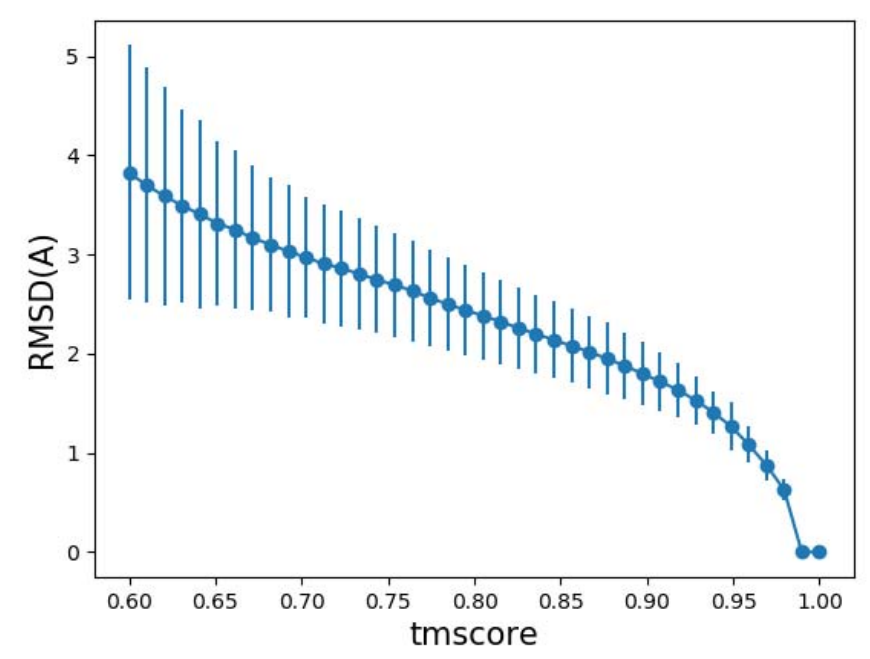

332 Figure 1. The relationship between the TM-score and the average RMSD of the

333 high-resolution training dataset. The average RMSD and the standard deviation of

334 RMSD decrease sharply as the TM-score increases. When TM-score $>0.4$. the average

335 RMSD is below $3 \AA$ and the standard deviation of RMSD is below $1 \AA$. 

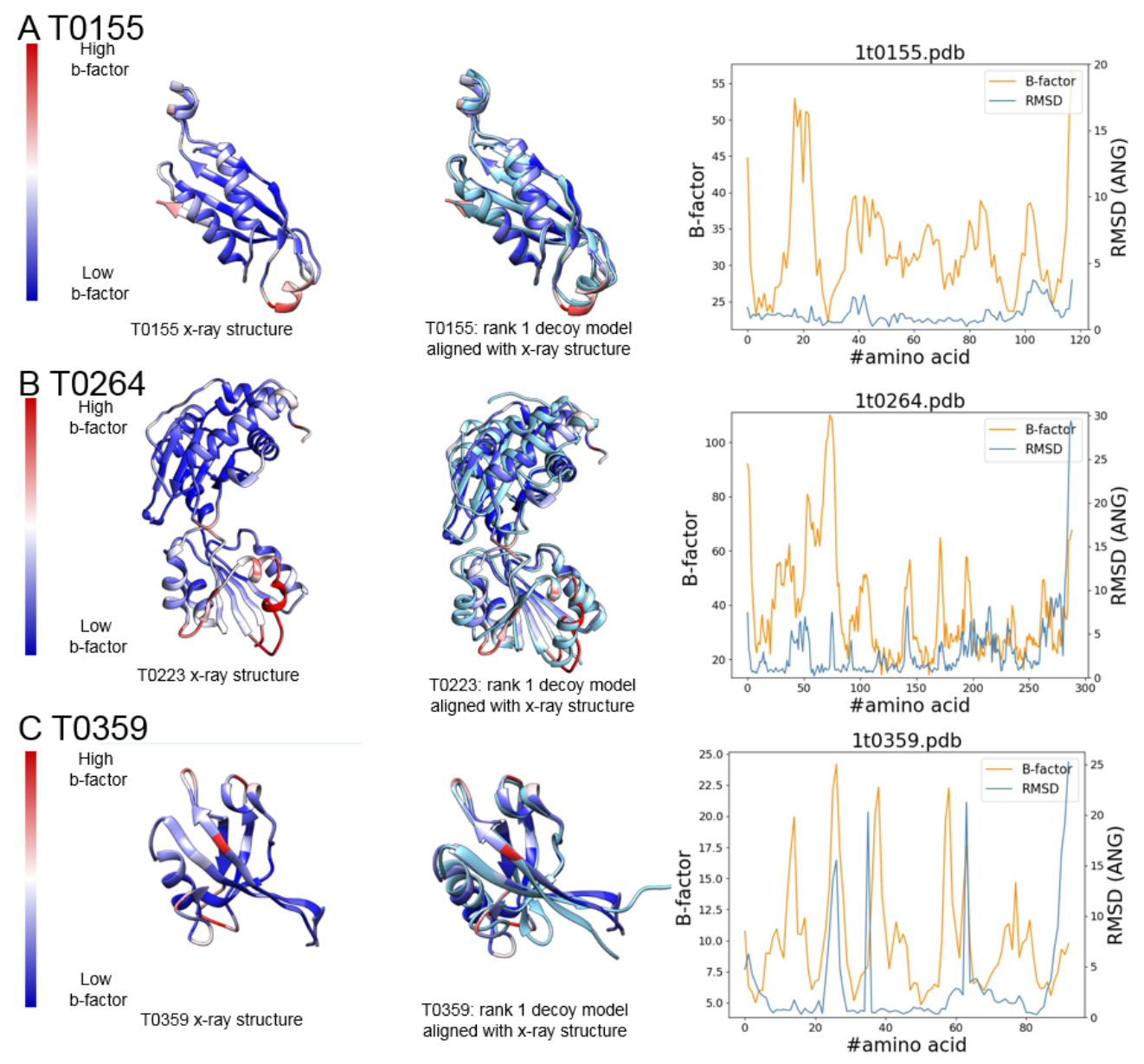

Figure 2. Relationship between $C_{\alpha}$ RMSD values and the corresponding B-factors

of the X-ray structures. a) For T0155, the rank 1 structure picked by PBE have tm-score of 0.919 with its X-ray structure. b) For T0264, the rank 1 structure picked by PBE have tm-score of 0.847 with its X-ray structure. c) For T0359, the rank 1 structure picked by PBE have tm-score of 0.774 with its X-ray structure. The structure deviation mainly locates in the loop region or the terminal region. 


\section{Method}

\section{In-house dataset}

348 The in-house dataset was obtained from the SCOP20 dataset ${ }^{1}$. The criteria for proteins

349 included in the in-house dataset are: 1) its structure is not determined by NMR, and 2)

350 the pair-wise sequence identity with those in independent test datasets is less than

$35120 \%$. Finally, 5,147 proteins were contained in our in-house dataset. And for each

352 protein, 400 random decoys were included using Rosetta de novo structure predictions

353 followed by all-atom refinement ${ }^{2}$.

354 The template modeling score (TM-score $)^{3}$ which measures the similarity of topologies

355 of two protein structures was used as the criteria. TM-score has a range between $(0,1]$

356 with better similarity having higher TM-scores. And the values of the decoys ranged

357 mainly between $0.2-0.4$, as shown in the extended data figure 2 .

358

359

360

361

362

363

364

365

366

367

\section{Independent datasets}

Several of the widely used decoy datasets were employed as the independent ones to evaluate the performance of our new PBE, including:

CASP5-8 dataset. The dataset was downloaded from https://zhanglab.ccmb.med.umich.edu/RW/ ${ }^{4}$. All of the 143 proteins' structures determined by X-ray and the corresponding decoy sets were remained for further use.

High resolution dataset. The high resolution dataset including 148 proteins' native structures and the decoy sets was downloaded from http://titan.princeton.edu/2010-10-11/HRdecoys/ ${ }^{5}$. In the present work, only 107 protein structures determined by X-ray and the corresponding decoy sets were 
remained for further use.

3D-Robot dataset. The 3D-Robot decoy dataset was generated by the 3DRobot, a program devoted for automated generation of diverse and well-packed protein structure decoys. It includes structural decoys of the Rosetta, I-TASSER and Modeller sets, and was from https://zhanglab.ccmb.med.umich.edu/3DRobot/decoys ${ }^{6}$. Among the proteins, 58, 39 and 19 X-ray determined structures and the corresponding decoy sets were remained for further use.

\section{Evolutionary information}

Each protein's evolutionary information was retrieved through applying the primary sequence to generate the position specific score matrix (PSSM) by running three iterations of PSI-BLAST against a non-redundant sequence database UniRef90 with an E-value cutoff of $0.001^{7}$. A 20-dimensinal vector $P$ representing probabilities of conservation against mutations to 20 different amino acids was returned for each residue in the protein. And for a protein with sequence length $L$, the PSSM can be represented as an $L^{*} 20$ matrix.

\section{Accuracy measures}

We use two criteria to evaluate the performance accuracy: 1) after comparing with the X-ray structure, we present the number of structures regarded as rank 1 and that of those regarded within rank 5 that have the X-ray structure (this is the criterion typically used); 2) we list the number of rank 1 structures within the TM-score thresholds. 
bioRxiv preprint doi: https://doi.org/10.1101/2020.04.23.056945; this version posted April 25, 2020. The copyright holder for this preprint (which was not certified by peer review) is the author/funder. All rights reserved. No reuse allowed without permission.

390 


\begin{tabular}{|c|c|c|}
\hline 391 & \multicolumn{2}{|c|}{ Reference } \\
\hline 392 & \multirow[t]{3}{*}{1} & Chandonia, J. M., Fox, N. K. \& Brenner, S. E. SCOPe: classification of large macromolecular \\
\hline 393 & & structures in the structural classification of proteins-extended database. Nucleic Acids Res $\mathbf{4 7}$ \\
\hline 394 & & D475-D481, doi:10.1093/nar/gky1134 (2019). \\
\hline 395 & \multirow[t]{3}{*}{2} & Simons, K. T., Bonneau, R., Ruczinski, I. \& Baker, D. Ab initio protein structure prediction of \\
\hline 396 & & CASP III targets using ROSETTA. Proteins-Structure Function and Bioinformatics, 171-176 \\
\hline 97 & & (1999). \\
\hline 398 & \multirow[t]{3}{*}{3} & Zhang, Y. \& Skolnick, J. Scoring function for automated assessment of protein structure \\
\hline 99 & & template quality. Proteins-Structure Function and Bioinformatics 57, 702-710, \\
\hline 00 & & doi:10.1002/prot.20264 (2004). \\
\hline 401 & \multirow[t]{3}{*}{4} & Zhang, J. \& Zhang, Y. A Novel Side-Chain Orientation Dependent Potential Derived from \\
\hline 02 & & Random-Walk Reference State for Protein Fold Selection and Structure Prediction. Plos One 5, \\
\hline 403 & & doi:ARTN e1538610.1371/journal.pone.0015386 (2010). \\
\hline 404 & \multirow[t]{3}{*}{5} & Rajgaria, R., McAllister, S. R. \& Floudas, C. A. Distance dependent centroid to centroid force \\
\hline 405 & & fields using high resolution decoys. Proteins-Structure Function and Bioinformatics 70, \\
\hline 406 & & 950-970, doi:10.1002/prot.21561 (2008). \\
\hline 407 & \multirow[t]{3}{*}{6} & Deng, H. Y., Jia, Y. \& Zhang, Y. 3DRobot: automated generation of diverse and well-packed \\
\hline 408 & & protein structure decoys. Bioinformatics 32, 378-387, doi:10.1093/bioinformatics/btv601 \\
\hline 409 & & (2016). \\
\hline 410 & \multirow[t]{5}{*}{7} & Schaffer, A. A. et al. Improving the accuracy of PSI-BLAST protein database searches with \\
\hline 411 & & composition-based statistics and other refinements. Nucleic Acids Res 29, 2994-3005, doi:DOI \\
\hline 412 & & 10.1093/nar/29.14.2994 (2001). \\
\hline 413 & & \\
\hline 414 & & \\
\hline & & \\
\hline
\end{tabular}


416

417

418

419

420

421

422

423

424

425

426

427

\section{Availability}

The program named "pickingnative" has been written in $\mathrm{C}++$ language and runs on the Linux platform. It is free of use on the web-server: www.166.111.152.74:8888/pbe_score/, and for academia user the executable code can be obtained after request by E-Mail: pan-xm@mail.tsinghua.edu.cn.

\section{Acknowledgments:}

Funding/Support: This work was supported in part by the project 39625008 from the National Natural Science Foundation of China.

Author Contributions: Dr Xian-ming and Xia-yu have full access to all of the data in the study and take responsibility for the integrity of the data and the accuracy of the analysis.

Study concept and design: Xian-ming and Xia-yu

Written all the computer programs: Xian-ming

Acquisition of data: Xian-ming, Lu-yun, and Xia-yu

Analysis and interpretation of data: Xian-ming, Xia-yu, Lu-yun

Drafting of the manuscript: Xian-ming, Xia-yu, Lu-yun

Critical revision of the manuscript for important intellectual content: Xian-ming

Statistical analysis: Xian-ming, Xia-yu, Luyun

Obtained funding: Xian-ming.

Administrative, technical, or material support: Xian-ming.

Study supervision: Xian-ming

All authors have read the final manuscript. 
438 Financial Disclosures: None reported.

439 Role of the Sponsors

440 The funding organizations did not have any role in the design and conduct of the study,

441 in the collection, management, analysis and interpretation of the data, or in the

442 preparation, review or approval of the manuscript.

443 Disclaimer

444 The content is only the responsibility of the authors.

445 Correspondence authors Dr. Xian-ming Pan, or Dr. Xia-yu Xia School of Life

446 Sciences, Tsinghua University, Beijing, 100084, China, Telephone: +86-10-62792827;

447 E-Mail: pan-xm@mail.tsinghua.edu.cn

448 


\section{Extended data}

450

\section{Extended Data Figure 1}

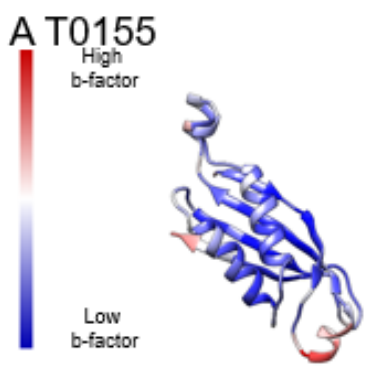

\section{BT0315}

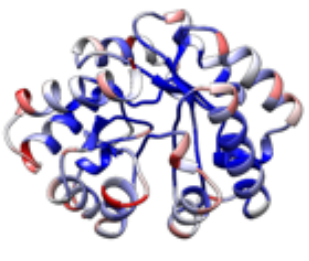

C T0423
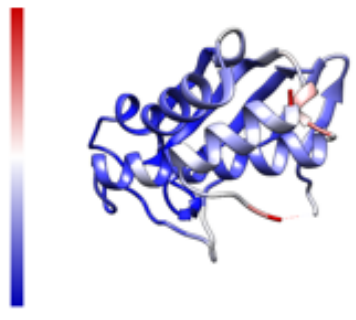
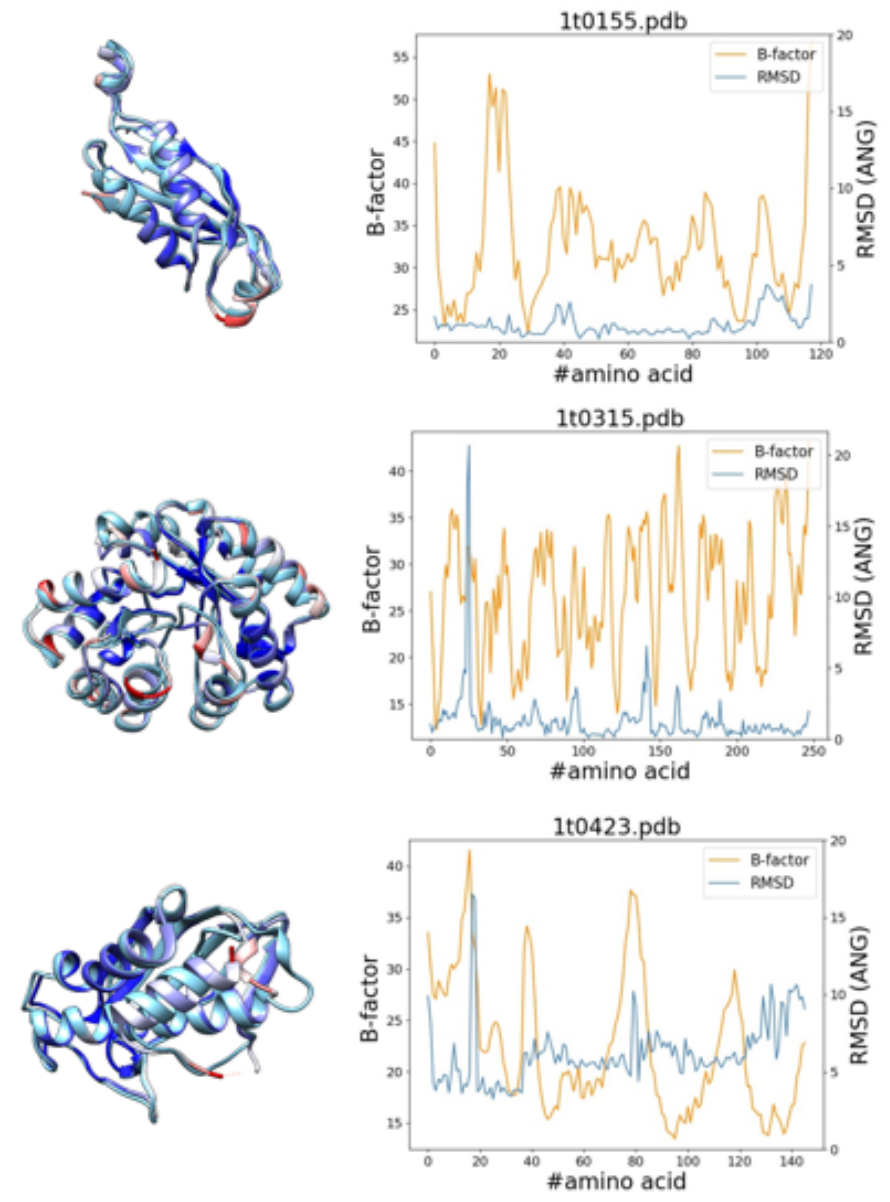
bioRxiv preprint doi: https://doi.org/10.1101/2020.04.23.056945; this version posted April 25, 2020. The copyright holder for this preprint (which was not certified by peer review) is the author/funder. All rights reserved. No reuse allowed without permission.
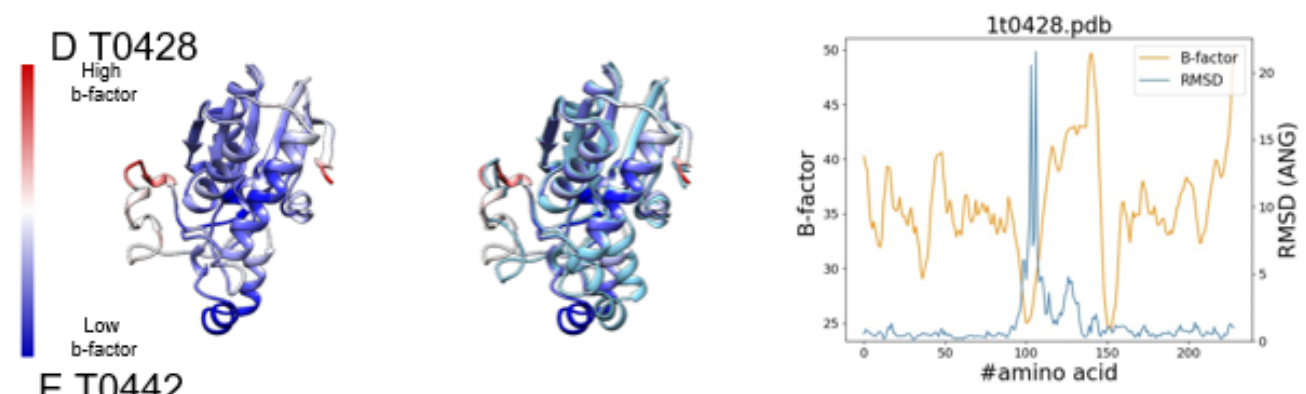

E T0442
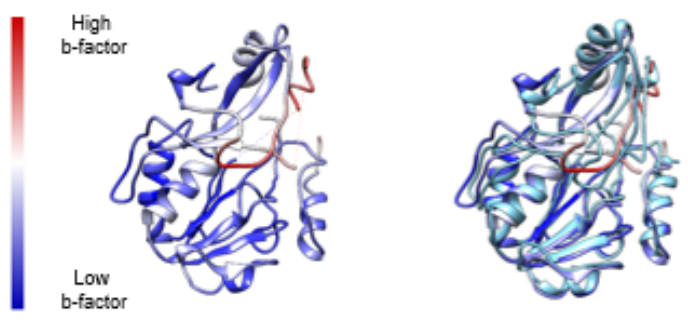

\section{F T0508}
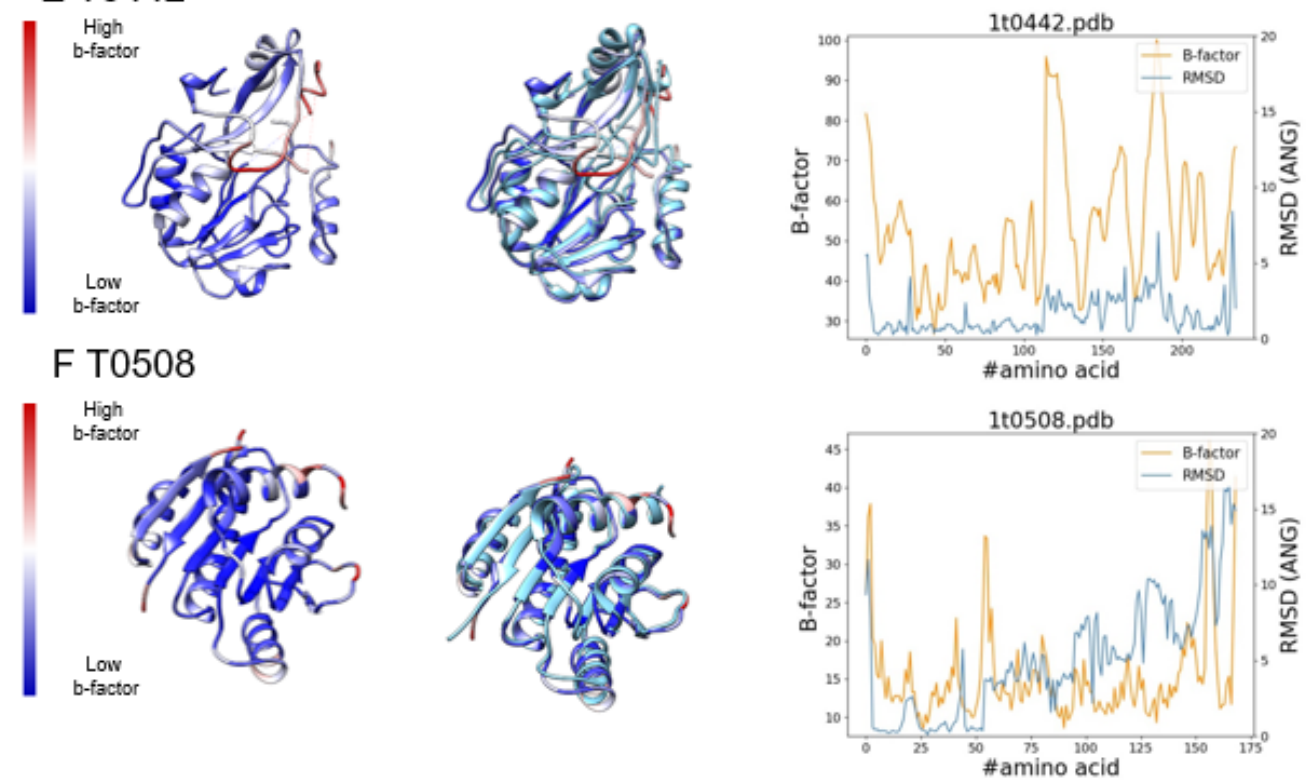

452 
bioRxiv preprint doi: https://doi.org/10.1101/2020.04.23.056945; this version posted April 25, 2020. The copyright holder for this preprint (which was not certified by peer review) is the author/funder. All rights reserved. No reuse allowed without permission.

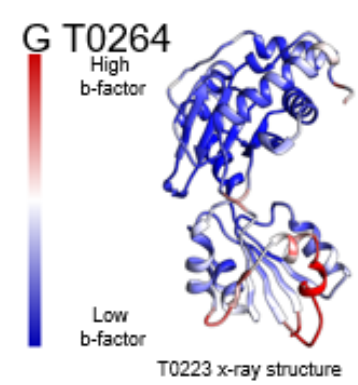

H T0380

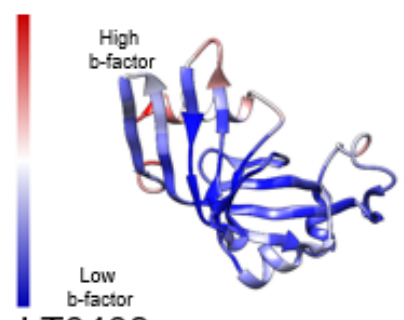

I T0408

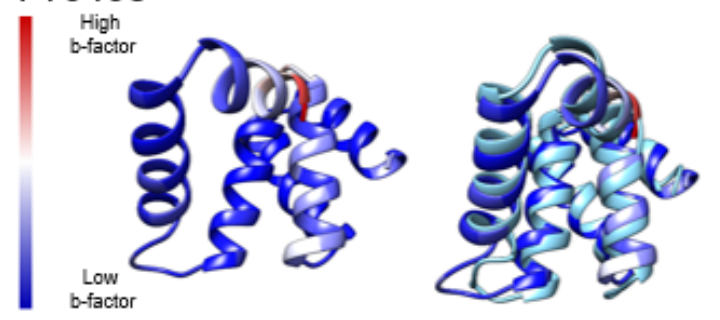

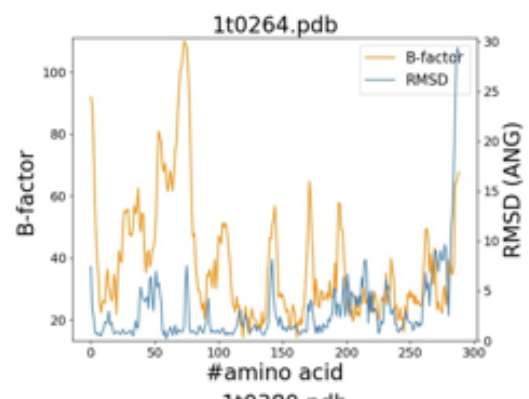
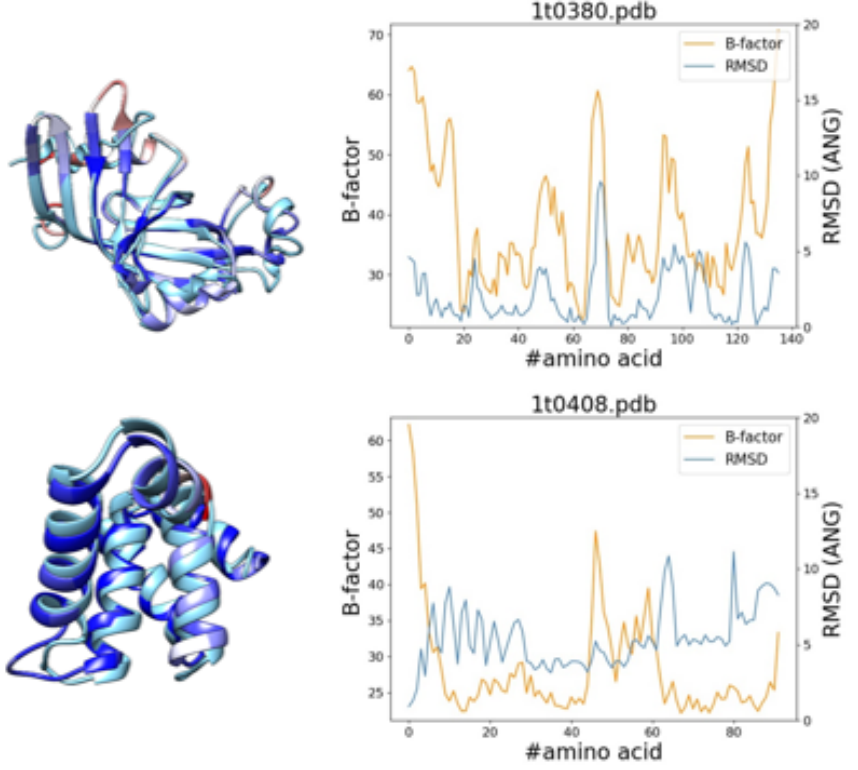


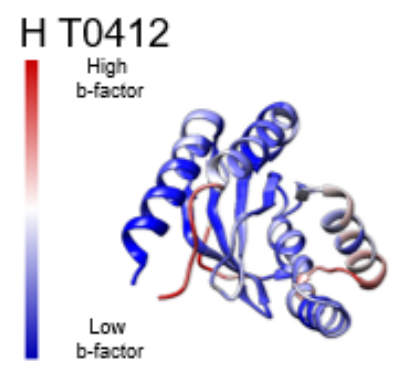

\section{K T0441}

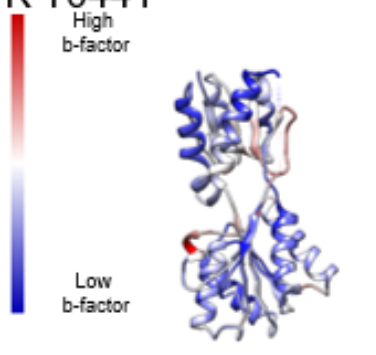

L T0445

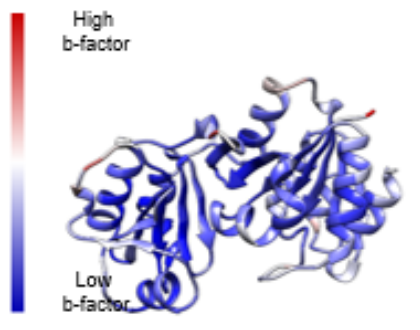

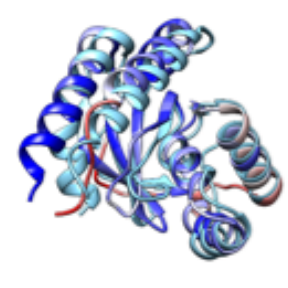
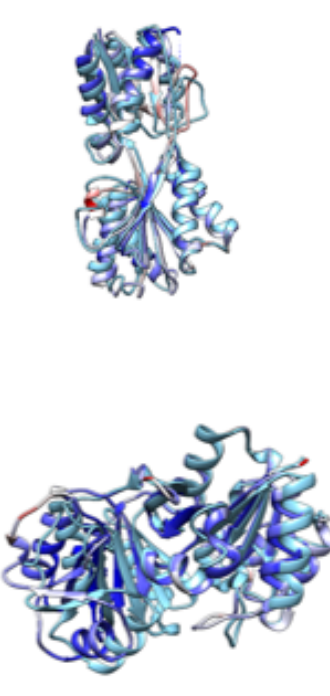
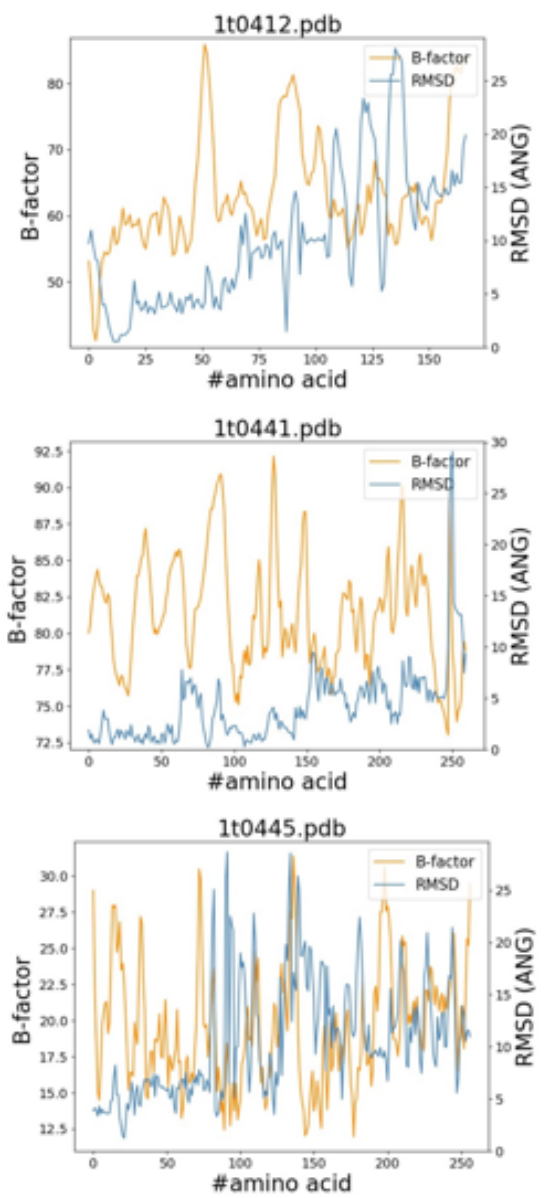
bioRxiv preprint doi: https://doi.org/10.1101/2020.04.23.056945; this version posted April 25, 2020. The copyright holder for this preprint (which was not certified by peer review) is the author/funder. All rights reserved. No reuse allowed without permission.
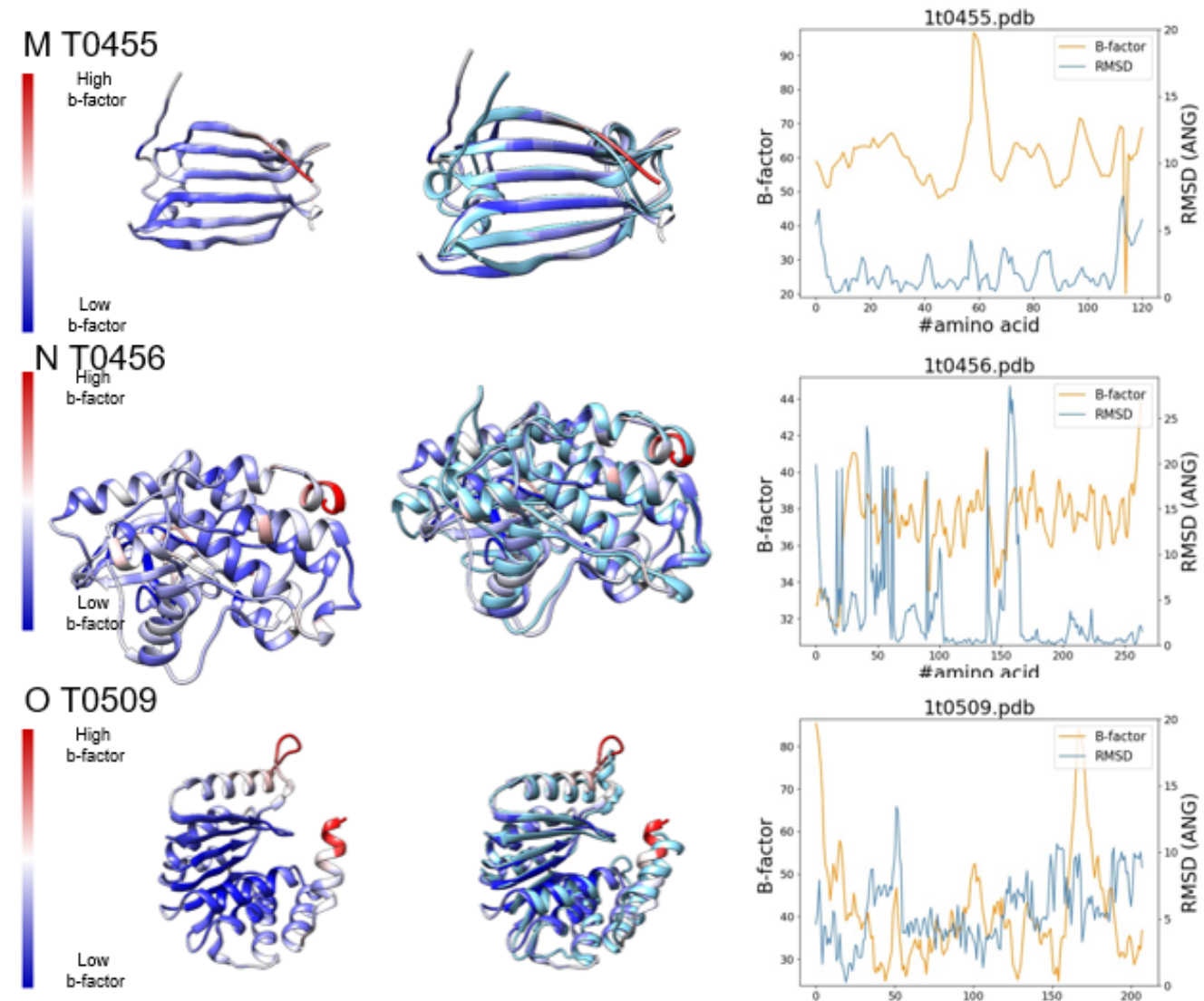

455

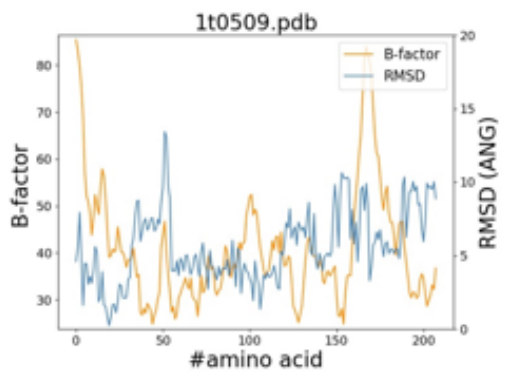


bioRxiv preprint doi: https://doi.org/10.1101/2020.04.23.056945; this version posted April 25, 2020. The copyright holder for this preprint (which was not certified by peer review) is the author/funder. All rights reserved. No reuse allowed without permission.

O T0359
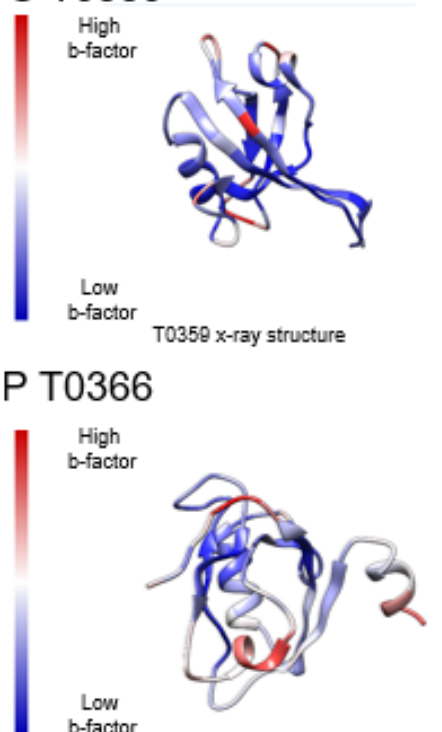

Q T0459

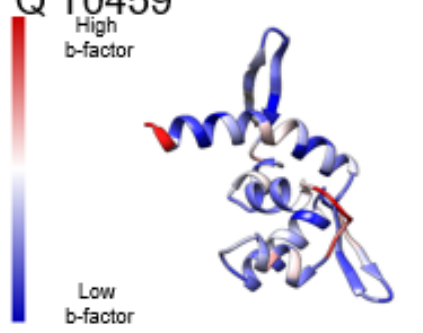

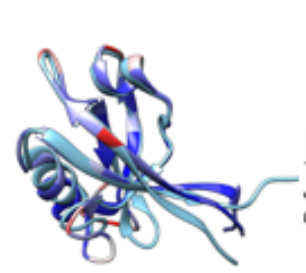

TO359: rank 1 decoy model aligned with $x$-ray structure
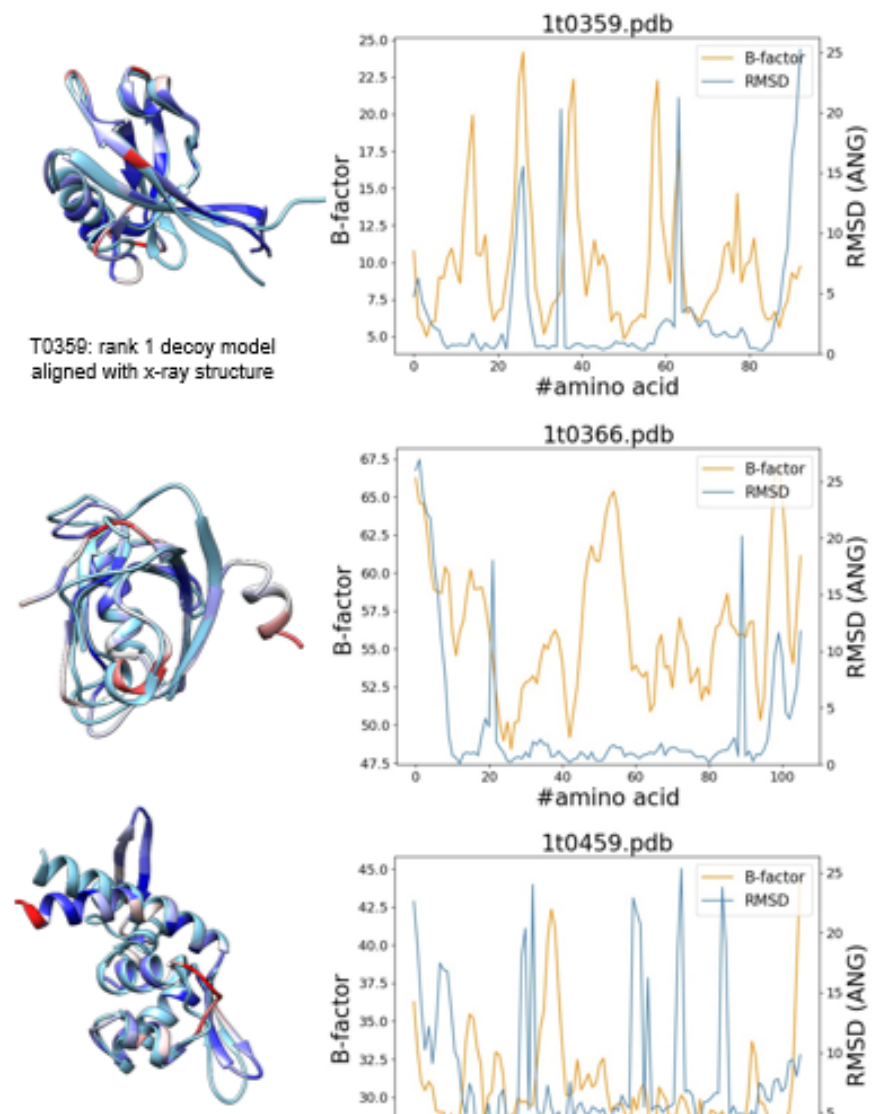

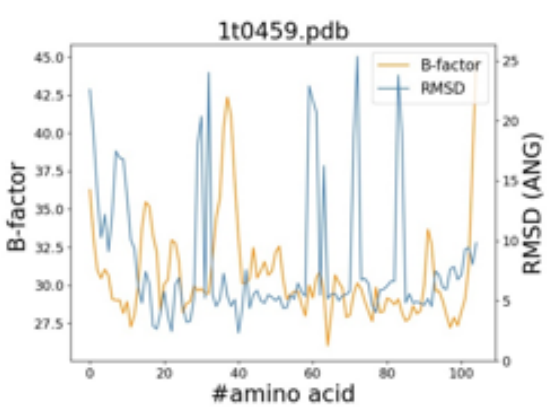



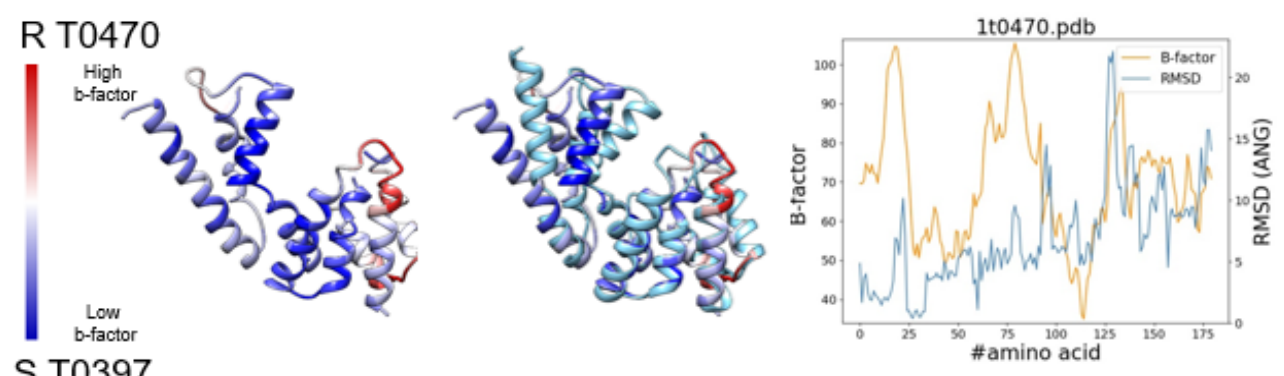

\section{S T0397}
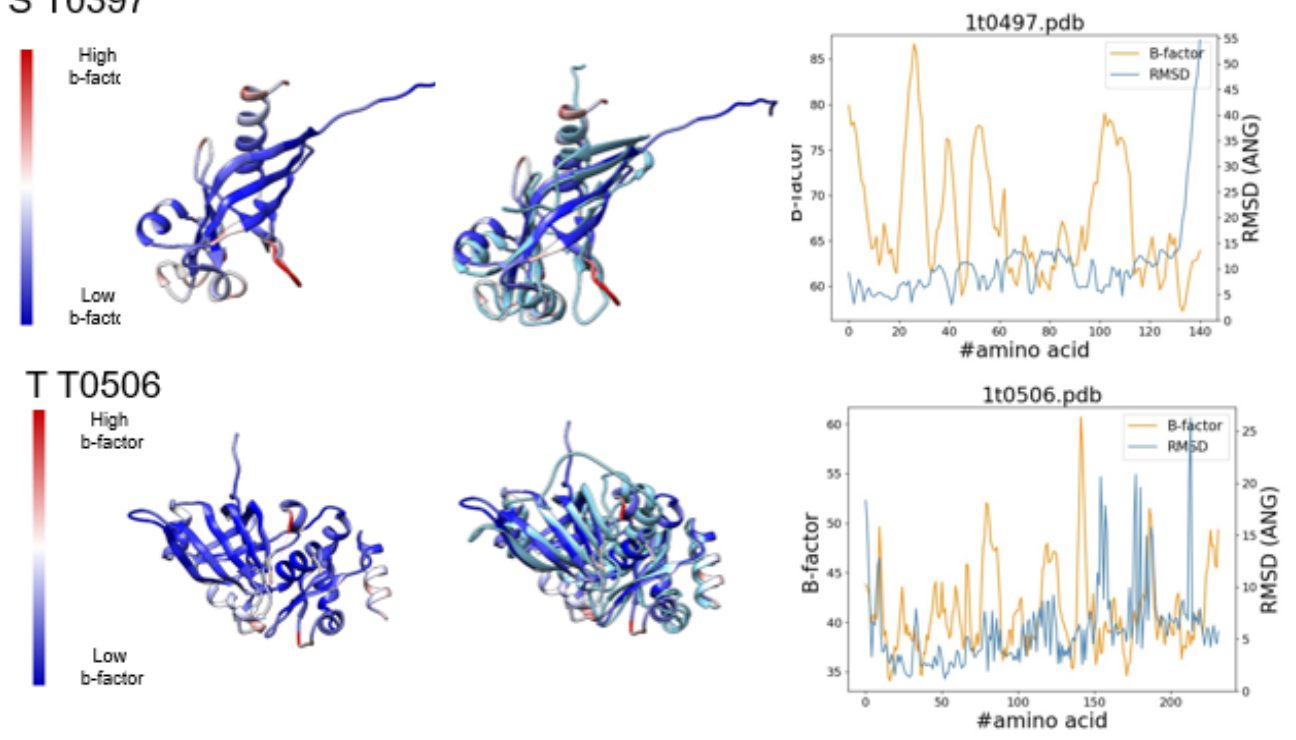

457

458 Extended Data Figure.1|. Relationship between $C_{\alpha}$ RMSD values and the

corresponding B-factors of the X-ray structures. 


\section{Extended Data Figure 2}

463

464

465

466

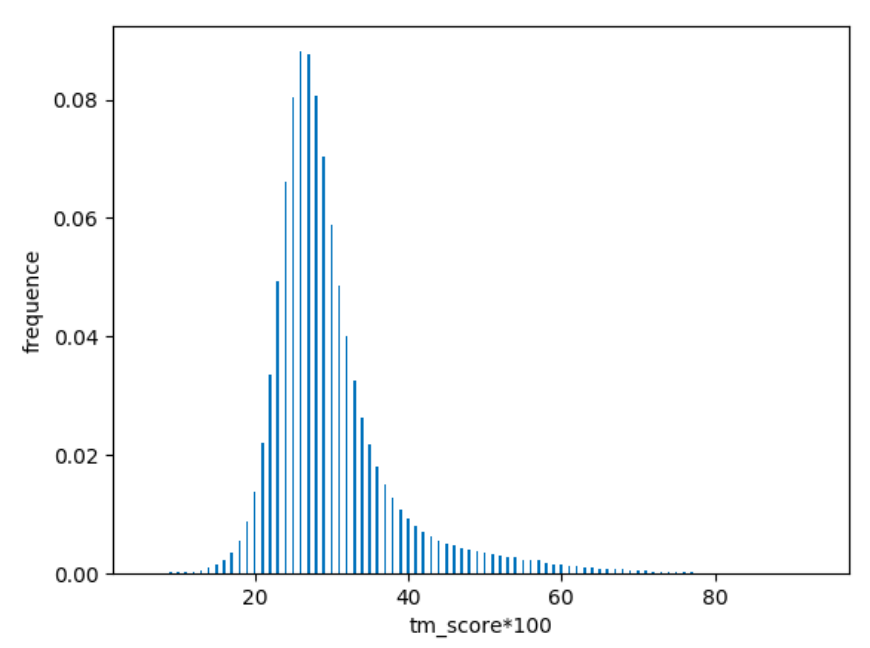

Extended Data Figure.2|. Distribution of the decoys' $t_{m}$ scores of the in-house

dataset. The decoys' TM-score mainly distribute in the range of 0.2-0.4 
467 Extended Data Table 1. Selecting accuracy of the PBE score on inhouse dataset.

\begin{tabular}{ccccc}
\hline \multirow{2}{*}{ TM-score } & \multicolumn{2}{c}{ Self-consistency } & \multicolumn{2}{c}{ Jackknife } \\
\cline { 2 - 5 } & Accuracy / \% & Z-score & Accuracy / \% & Z-score \\
\hline $1.0(x-$-ray) & 100.0 & -9.67 & 98.62 & -9.00 \\
\hline$>0.9$ & 100.0 & -9.67 & 98.62 & -9.00 \\
\hline$>0.8$ & 100.0 & -9.33 & 98.97 & -8.69 \\
\hline$>0.7$ & 100.0 & -8.04 & 99.26 & -7.49 \\
\hline$>0.6$ & 100.0 & -6.20 & 99.53 & -5.79 \\
\hline$>0.5$ & 100.0 & -4.37 & 99.57 & -4.09 \\
\hline
\end{tabular}

